\title{
Review Article \\ Physicochemical Properties of Nanomaterials: Implication in Associated Toxic Manifestations
}

\author{
Manzoor Ahmad Gatoo, ${ }^{1}$ Sufia Naseem, ${ }^{1}$ Mir Yasir Arfat, ${ }^{1}$ Ayaz Mahmood Dar, \\ Khusro Qasim, ${ }^{3}$ and Swaleha Zubair ${ }^{4}$ \\ ${ }^{1}$ Department of Biochemistry, Jawaharlal Nehru Medical College, Aligarh Muslim University, Aligarh, Uttar Pradesh 202002, India \\ ${ }^{2}$ Department of Chemistry, Aligarh Muslim University, Aligarh, Uttar Pradesh 202002, India \\ ${ }^{3}$ Department of Mechanical Engineering, Aligarh Muslim University, Aligarh, Uttar Pradesh 202002, India \\ ${ }^{4}$ Women's College, Aligarh Muslim University, Aligarh, Uttar Pradesh 202002, India
}

Correspondence should be addressed to Swaleha Zubair; swalehazubair@yahoo.com

Received 14 May 2014; Accepted 16 June 2014; Published 6 August 2014

Academic Editor: Mohammad Owais

Copyright (C) 2014 Manzoor Ahmad Gatoo et al. This is an open access article distributed under the Creative Commons Attribution License, which permits unrestricted use, distribution, and reproduction in any medium, provided the original work is properly cited.

\begin{abstract}
Nanotechnology has emerged as one of the leading fields of the science having tremendous application in diverse disciplines. As nanomaterials are increasingly becoming part of everyday consumer products, it is imperative to assess their impact on living organisms and on the environment. Physicochemical characteristics of nanoparticles and engineered nanomaterials including size, shape, chemical composition, physiochemical stability, crystal structure, surface area, surface energy, and surface roughness generally influence the toxic manifestations of these nanomaterials. This compels the research fraternity to evaluate the role of these properties in determining associated toxicity issues. Reckoning with this fact, in this paper, issues pertaining to the physicochemical properties of nanomaterials as it relates to the toxicity of the nanomaterials are discussed.
\end{abstract}

\section{Introduction}

Nanotechnology is being considered as the next step logical in integrating technology based science with other sister disciplines including biology, chemistry, and physics [1]. Royal Society and Royal Academy of Engineering have defined "nanoscience" as the study of phenomena and manipulation of materials at atomic, molecular, and macromolecular scales while nanotechnology has been defined as the design, characterization, production, and application of structures, devices and systems by controlling shape and size at nanometre scale [2]. Current nanotechnology is the building device of microscopic or even molecular size, which will potentially be benefiting medicine, environmental protection, energy, and space exploration [3-6]. In the last few years, the term "nanotechnology" has been inflated and has almost become synonymous for objects that are innovative and highly promising [5, 7-9]. A more generalized description of nanotechnology could be manipulation of matter with at least one dimension of size from 1 to 100 nanometres, namely, nanomaterials. Intriguingly, these nanomaterials embody distinctive physicochemical and biological properties compared to their conventional counter parts which endow them their beneficial characteristics.

In the recent scenario, researches engrossing different nanoparticles are evolving at a tremendous pace owing to which engineered nanomaterials (ENMs) are increasingly becoming part of daily life in the form of cosmetics, food packaging, drug delivery, therapeutics, biosensors, and so forth and, with these, unprecedented avenues for exposure of nanoparticles (NPs) to environment and living beings are increasing [10]. The increasing exposure of nanomaterials makes it imperative to assess the toxic effect of nanoparticle based materials; moreover, as the physical and chemical characteristics of nanomaterials influence the properties of nanoparticles, it is also more imperative to evaluate the physicochemical properties of nanomaterials including size, surface area, solubility, chemical composition, shape, agglomeration state, crystal structure, surface energy, surface charge, surface morphology, and surface coating and also role 
of individual characteristic property in imparting toxic manifestations. Reckoning with these facts, in this review, an attempt has been made to analyse the corelation of these physicochemical properties with the toxicity of engineered nanomaterials.

It is in general consensus that nanoparticles exhibit toxic manifestations through diverse mechanisms and can result in allergy, fibrosis, organ failure, nephrotoxicities, haematological toxicities, neurotoxicities, hepatological toxicities, splenic toxicities, and pulmonary toxicities, among others [11-14].

\section{Physicochemical Properties of Nanoparticles and Their Effect on Toxicity}

As a matter of fact, nanomaterials have unique properties relative to bulk counterpart which impart them beneficial characteristics; ironically, they may also bestow them with unique mechanisms of toxicity. In general, toxicity has been thought to originate from nanomaterials' size and surface area, composition, shapes, and so forth as reviewed in the following sections.

2.1. Size and Surface Area of the Particles. Particle size and surface area play a major role in interaction of materials with biological system. Seemingly, decreasing the size of the materials leads to an exponential increase in surface area relative to volume, thereby making the nanomaterial surface more reactive on itself and to its contiguous milieu. Of note, particle size and surface area dictate how the system responds to, distributes, and eliminates the materials [15]. It has been established that various biological mechanisms including endocytosis, cellular uptake, and efficiency of particle processing in the endocytic pathway are dependent on size of the material $[12,16]$. Various researchers have evaluated in vitro cytotoxicity of NPs of different size employing various cell types, culture conditions, and exposure times [17, 18]; however, their in vivo evaluation is difficult owing to their more complex nature in the biological systems and requires more comprehensive understanding of the particles [19], though various authors have evaluated their toxicity issues in biological systems employing various in vivo models. In general, the size dependent toxicity of nanoparticles can be attributed to its ability to enter into the biological systems [20] and then modify the structure of various macromolecules [21], thereby interfering with critical biological functions.

One of the major mechanisms for in vivo toxicity of the ENMs is through the generation of oxidative responses by formation of free radicals, in which size has a decisive role to play as highlighted by many authors that the smaller the size the more able it is towards formation of ROS. These free radicals have been known to impart hazards to biological systems mainly through DNA damage, through oxidation of lipids, and by ensuing of inflammatory responses.

Furthermore, several studies employing diverse class of nanoparticles showed that surface area is also critical factor in displaying toxic manifestations (lung and other epithelial-induced inflammatory responses) in rodents [22].
With decrement in size of nanoparticles, surface area increases which causes a dose dependent increment in oxidation and DNA damaging abilities of these nanomaterials [23] much higher than larger particles with the same mass dose [24].

Nanoparticle size also dictates their pharmacological behaviours. It has been observed that NPs smaller than $50 \mathrm{~nm}$ (administrated by intravenous injection) transverse quickly to nearly all tissues and impart potentially toxic manifestations in various tissues; on the other hand, NPs greater than $50 \mathrm{~nm}$ (in particular $100-200 \mathrm{~nm}$ positively charged particles) are readily taken up by RES which refrain their path to other tissues [25]. Although the clearance by reticuloendothelial system (RES) safeguard other tissues, it makes RES organs such as the liver and spleen as main targets of oxidative stress.

Several toxicological studies have demonstrated that smaller nanoparticles of dimensions $<100 \mathrm{~nm}$ cause adverse respiratory health effects compared to larger particles of the same material $[24,26]$. Inhaled particles of different sizes exhibit different fractional depositions within human respiratory tract. It has been observed that ultrafine particles with diameters $<100 \mathrm{~nm}$ deposits in all regions, whereas particles $<10 \mathrm{~nm}$ deposits in the tracheobronchial region, while particles between 10 and $20 \mathrm{~nm}$ deposits in the alveolar region [27]. As a result, the translocation or distribution of NPs has been found to be size dependent, which in turn decide their toxicities issues.

Kreyling et al. [28] showed that instillation of Ir192particles of $80 \mathrm{~nm}$ resulted in accumulation in the rat liver with an extent of $0.1 \%$ of total amount, while particles of $15 \mathrm{~nm}$ size displayed increased accumulation to an extent of $0.3-0.5 \%$. Moreover, it has been observed that when smaller particles are retained in the respiratory tract for longer duration it leads to increased translocation to the pulmonary interstitium with impairment of alveolar macrophages function. Redistribution of NPs from their site of deposition [29] or deposition into renal tissues and escape from normal phagocytic defences [30] may also lead to toxicity.

Moreover, size of nanoparticles also influences their oral toxicity. In general, the oral toxicity increases with decreasing size. In one of the studies, it was observed that oral toxicity of copper nanoparticles increased with decreasing size. More importantly, larger particles were nontoxic even at higher doses, whereas smaller particles were moderately toxic [31].

Furthermore, employing zebrafish as a model to evaluate the in vivo toxicity of different gold and silver nanoparticles in the size range of $3,10,50$, and $100 \mathrm{~nm}$, the researchers reported that AgNPs produce size dependent mortality, whereas, interestingly but not surprisingly, the behaviour of $\mathrm{Au}$ NPs was independent of size [31]. Moreover, in concordance with this study, a similar correlation was observed for the large-sized cyanoacrylate nanoparticles, in which toxicity was dependent on the chemical properties and molecular chain length and was independent of particle size [32]; however vice-versa was true in case of small-sized polyacrylate nanoparticles, wherein toxic manifestations were independent of chemical chemistries. 
It implies that although size and surface area are important factors in determining toxicity of nanoparticles other factors such as chemical nature of the constituents may also contribute to the intrinsic toxicity of the nanoparticles.

2.2. Effect of Particle Shape and Aspect Ratio. There has been flurry of major advancement in the understanding of interplay between particle size and shape for development of more efficacious nanomaterial based targeted delivery system; nevertheless, this also reenforces that their untoward effects should also be examined. As well depicted in Figure 1, nanomaterials come in varied shapes including fibres, rings, tubes, spheres, and planes.

Shape dependent toxicity has been reported for myriads of nanoparticles including carbon nanotubes, silica, allotropies, nickel, gold, and titanium nanomaterials [3336]. Basically, shape dependent nanotoxicity influences the membrane wrapping processes in vivo during endocytosis or phagocytosis [37]. It has been observed that endocytosis of spherical nanoparticles is easier and faster as compared to rod shaped or fibre like nanoparticles [38] and more importantly spherical nanoparticles are relatively less toxic irrespective of whether they are homogenous or heterogeneous [39]. Nonspherical nanomaterials are more disposed to flow through capillaries causing other biological consequences [40]. Studies have shown that rod shaped SWCNT can block $\mathrm{K}^{+}$ion channels two to three times more efficiently than spherical carbon fullerenes [41]. Of note, theshape dependent toxicity of silica allotropies is evident by fact that amorphous silica is used as food additive while as crystalline silica is suspected human carcinogen [33]. Similarly, it has been shown that uptake of gold nanorods is slower than spherical nanospheres [35] and uptake of nanorods reaches maximum when aspect ratio approaches unity [42]. It has been observed that $\mathrm{TiO}_{2}$ fibres are more cytotoxic than spherical entities [43].

Moreover, it has also been observed that the higher the aspect ratio, the more the toxicity of particle [44]. In case of asbestos induced toxicity, it was observed that asbestos fibres longer than 10 microns caused lung carcinoma while fibres $>5$ microns caused mesothelioma and fibres $>2$ microns caused asbestosis [45] as longer fibre will not be effectively cleared from the respiratory tract due to the inability of macrophages to phagocytise them. Hamilton et al. [36] showed that $\mathrm{TiO}_{2}$ fibers with a length of $15 \mathrm{~mm}$ are highly toxic compared to fibers with a length of $5 \mathrm{~mm}$ and initiate an inflammatory response by alveolar macrophages in mice. The toxicity of fibres with long aspect is closely related to their plasma shelf life. The fibres that are sufficiently soluble in lung fluid can disappear in a matter of months, while the insoluble fibers are likely to remain in the lungs indefinitely. It was also observed that long-aspect ratio particles (SWCNTs) produce significant pulmonary toxicity compared to spherical particles [46]. Further, long MWCNTs cause inflammation of the abdominal wall after inta-abdominal instillation, while no inflammatory responses were observed in case of short MWCNT [47]. Accordingly, as the intricacies of these phenomena increasingly unravel, they would certainly help towards implementation of safer nanotechnology based systems.
2.3. Effect of Surface Charge. Surface charge also plays an important role in toxicity of nanoparticles as it largely defines their interactions with the biological systems. Various aspects of nanomaterials such as selective adsorption of nanoparticles [48], colloidal behaviour, plasma protein binding [49], bloodbrain barrier integrity, and transmembrane permeability are primarily regulated by surface charge of nanoparticles [50]. Of note, positively charged nanoparticles show significant cellular uptake compared to negatively charged and neutral nanoparticles, owing to their enhanced opsonization by the plasma proteins. Moreover, they have also been shown to induce hemolysis and platelet aggregation [51] owing to which causes severe toxicity to the system.

As surface charge is a major determinant of colloidal behaviour, it specifically influences the organism response upon exposure to nanoparticles by changing their shape and size through aggregate or agglomerate formation [48]. For example the toxicity of dendrimers is influenced by surface charge and it has been observed that positively charged PAMAM dendrimers (G4) exhibit time-dependent toxicity toward zebrafish and mice embryos while anionic PAMAM dendrimers display no toxic manifestations [52]. Similarly positively charged Si nanoparticles $\left(\mathrm{Si}-\mathrm{NP}-\mathrm{NH}_{2}\right.$ ) have been shown to be more cytotoxic compared to neutral and negatively charged Si nanoparticles which display minimal to no cytotoxicity issues [53]. Pietroiusti et al. found that acid functionalized SWCNTs exhibits marked embryo toxic effect compared to pristine SWCNTs in pregnant mice models [49].

It has also been observed that surface charge of nanoparticles alters blood-brain barrier integrity and transmembrane permeability. In this regard, it was found that the negatively charged NPs in the size range of 50 to $500 \mathrm{~nm}$ permeate skin after dermal administration, whereas no such effects were seen for positively charged and neutral particles irrespective of their sizes. Basically, NPs of $50 \mathrm{~nm}$ permeate the skin due to the small size and large specific surface area, whereas $500 \mathrm{~nm}$ particles permeate the skin because the high number and density of charged groups lead to a high charge concentration that overcomes the skin barrier [54].

As the interactions of NPs with the biological systems are largely influenced by their surface charge, the research fraternities have employed various amendments to shield or modulate their surface characteristics so as to reduce their toxic manifestations, a glimpse of which has been provided in the later part of the paper.

\subsection{Effect of Composition and Crystalline Structure. Although} it has been emphasized that particle size plays significant role in deciding toxicity of nanoparticles, we cannot simply ignore studies exemplifying comparable toxicities for diverse nanoparticles chemistries having the same dimensions. These studies highlight that the composition and crystalline structure of nanoparticles also influence their toxicity issues. In a study by Griffitt et al. [55] using zebrafish, daphnids, and algal species as models of various trophic levels it was observed that nanosilver and nanocopper with their soluble forms caused toxicity in all tested organisms, whereas $\mathrm{TiO}_{2}$ of the same dimensions did not cause any toxicity issues [55], 


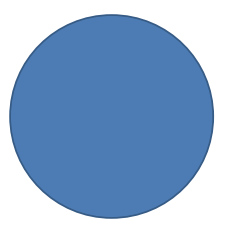

Spherical

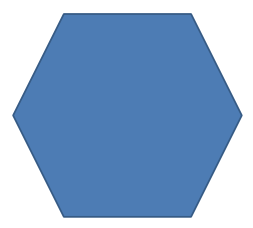

Hexagonal

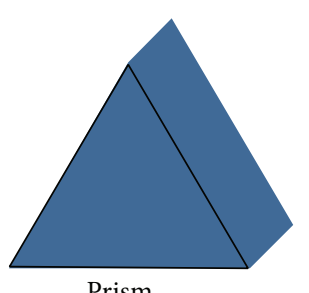

Prism

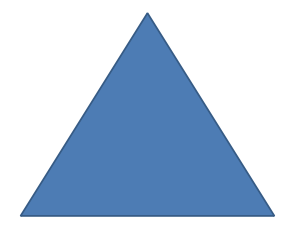

Triangular

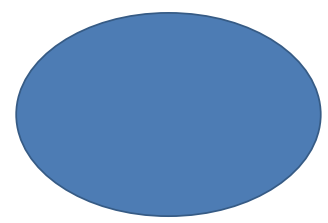

Oval
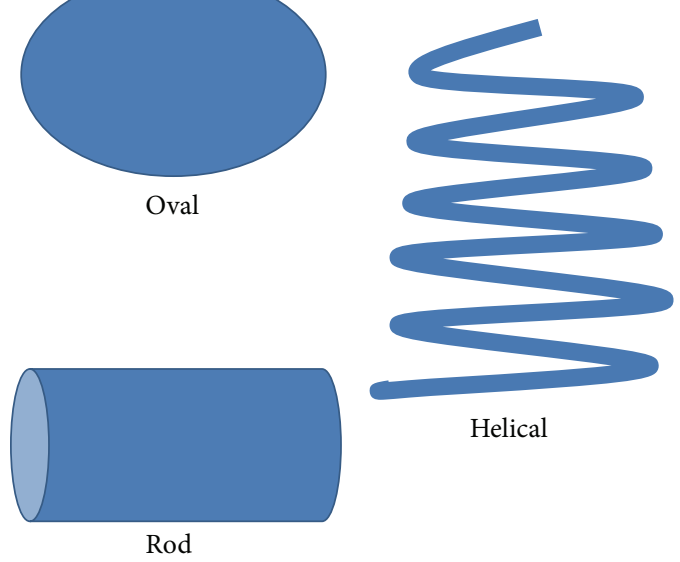

Helical

FIGURE 1: Various shapes of nanoparticles.

thus emphasizing role of compositions in determining the toxicities of NPs.

Crystal structure also influences the toxicity of nanoparticles and it has been observed that rutile $\mathrm{TiO}_{2}$ nanoparticles induce oxidative DNA damage, lipid peroxidation, and micronuclei formation in the absence of light, whereas anatase nanoparticles of the same size and chemical composition did not [26]. Besides, nanoparticles can change crystal structure after interaction with water or other dispersion medium. It has been reported that $\mathrm{ZnS}$ nanoparticles become more ordered in the presence of water by rearranging their crystal structure and become more close to the structure of a bulk piece of solid $\mathrm{ZnS}$ [56], thereby embarking that the solvent also has a role in the manifestations of toxicities displayed by the nanoparticulate systems as detailed later in the text.

2.5. Effect of Aggregation and Concentration. The aggregation states of nanoparticles also influence their toxicities. Basically, the aggregation states of NPs depend on size, surface charge, and composition among others. It has been observed that carbon nanotubes are mainly accumulated in liver, spleen, and lungs without manifesting any acute toxicity but induce cytotoxic effects mostly because of accumulation of aggregates for longer periods [57]. Agglomerated carbon nanotubes have more adverse effects than well-dispersed carbon nanotubes and enhance the pulmonary interstitial fibrosis [58]. Moreover, generally, it has been observed that with increase in the concentration of nanoparticles, the toxicity decreases at higher concentration.
2.6. Effect of Surface Coating and Surface Roughness. The surface properties of particles have significant role on toxicity of nanoparticles as they play a critical role in determining the outcome of their interaction with the cells and other biological entities. Surface coating can affect the cytotoxic properties of nanoparticles by changing their physicochemical properties such as magnetic, electric, and optical properties and chemical reactivity $[17,59]$ and can alter the pharmacokinetics, distribution, accumulation, and toxicity of nanoparticles. It has been known that the presence of oxygen, ozone, oxygen radicals and transition metals on nanoparticle surfaces leads to the generation of ROS and the induction of inflammation by these systems $[23,24,60]$; these certainly influence their associated toxicities issues. To this end, more specifically, Fubini et al. [61] have shown that the specific cytotoxicity of silica is strongly associated with the occurrence of surface radicals and reactive oxygen species on their surfaces.

However, on the other side of coin, surface coating could also be employed to reduce the toxicity issues of the nanoparticles. In general, surface coating can mitigate or eliminate the adverse effects of nanoparticles. In particular, proper surface coating can lead to stabilization of nanoparticles as well as elude release of toxic ions from nanomaterials [62].

To this end, surface modifications of NPs employing hydrophilic and flexible polyethylene glycol (e.g., pegylation) and other surfactant copolymers (e.g., poloxamers and polyethylene) have been considerably used by the research fraternity off late in this advancing field of nanotechnology to stabilize nanoparticulate systems in biological milieu. 
Although PEG imparts long circulatory time to the nanoparticulate systems mainly by stabilising them in biological system, they could not be indiscriminately used and, more importantly, they have to be chosen with caution, as studies have shown that particles coated with lower molecular weight PEG were quickly eliminated from circulation after injection, whereas QDs coated with high molecular weight remained in the blood circulation for longer time [63].

Surface coatings are important for QDs to render them nontoxic as metallic core of QDs is hydrophobic and is composed of heavy toxic metals like cadmium. In general, secondary coating is needed to increase the QD core's durability, prevent ion leaching, and increase water dispersibility [64]. However, care should be takento choose appropriate coating agents, as weaker surface coatings are prone to oxidative or photolytic degradation leading to exposure of the metalloid core, which may be toxic or can pave the way for unforeseen reactions inside the body [65]. Intriguingly, Chen and Gerion [66] developed silanized QDs (QDs coated with silica) embodying attributes of lack of genotoxicity issues owing to their least interaction with proteins and DNA. Moreover, various biocompatible polymers have also been widely used as coating materials for SPIONs to avoid their toxicity issues [67].

Furthermore, in selecting the appropriate coating material, charge of the coating agent should also be considered. As already discussed that the charge of nanoparticles plays important role in influencing their toxic behaviours, on this line, it has been observed that QDs coated with negatively charged serum protein albumin show a higher liver uptake and faster blood clearance relative to the QDs without albu$\min [68,69]$. Coatings and functionalization can also reduce the in vivo toxicity of carbon nanotubes [70]. Moreover, it has also been demonstrated that spherical gold nanoparticles with various surface coatings have been found to be nontoxic to human cells $[71,72]$.

Furthermore, as the attributes of nanoparticles such as surface roughness, hydrophobicity, and charge of nanoparticles influence the phenomena of cellular uptake of nanoparticles [73], they indeed influence the toxicity associated with nanoparticles. Surface coarseness dictates the strength of nanoparticle-cell interactions and promotes cell adhesion. Pore structure is critical in cell-nanoparticle interactions. It has been demonstrated that size dependent hemolysis effect of mesoporous silica nanoparticles is only observed when the nanoparticles have long range ordered porous structure $[74,75]$. De Angelis et al. [75] showed that nanoporous silicon NPs with a pore size of about $2 \mathrm{~nm}$ do not have any toxicity in mouse-models with no histological evidence of tissue pathology. Similarly Park et al. [76] observed that luminescent porous silicon nanoparticles did not show any toxicity in animal models.

2.7. Effect of Solvents/Media. Medium/solvent conditions have been known to affect particle dispersion and agglomeration state of nanoparticles, which in turn have effect on their particle size, thereby influencing the toxicity associated with nanoparticles. It has been observed that particles of
$\mathrm{TiO}_{2}, \mathrm{ZnO}$, or carbon black have significantly greater size in PBS than in water; moreover, it is also in general consensus that NPs display different diameters in biological milieu $[77,78]$. Accordingly, the toxic effects of nanoparticles show variation depending upon the medium composition in which the nanoparticles are suspended; in another way round, the same nanoparticles exhibit different toxic manifestations when dissolved in different mediums [79, 80]. Although, the dispersing agent may improve the physicochemical and solution properties of nanomaterials formulations, they may also adversely affect the toxicity of nanomaterials.

\section{Conclusion}

Nanotechnology is being envisaged as burgeoning field with many potential human health benefits and with rapid upsurge in the field; it becomes increasingly imperative to evaluate the toxicities issues associated with these nanomaterial based products.

While the toxicity of bulk materials is affected mainly by their composition, however, in case of nanomaterials, additional physicochemical properties such as size, surface area, surface chemistry, surface roughness, dispersion medium, and ability to agglomerate play vital role in determining their toxicity. With newer nanomaterials based products being introduced in the market on daily bases, there is urgent need to reduce the knowledge gap between the physicochemical properties and their influence on the manifestation of toxicities issues. This will certainly pave ways towards maneuvering these physicochemical properties for their safer implementation in diverse fields.

\section{Conflict of Interests}

The authors declare that there is no conflict of interests regarding the publication of this paper.

\section{References}

[1] J. M. Lehn, "Toward self-organization and complex matter," Science, vol. 295, no. 5564, pp. 2400-2403, 2002.

[2] K. Aslan, I. Gryczynski, J. Malicka, E. Matveeva, J. R. Lakowicz, and C. D. Geddes, "Metal-enhanced fluorescence: an emerging tool in biotechnology," Current Opinion in Biotechnology, vol. 16, no. 1, pp. 55-62, 2005.

[3] C. Raison, "Gold nanoparticle-based diagnostic test for rapid diagnosis of leading infectious diseases," Expert Review of Molecular Diagnostics, vol. 13, no. 3, article 230, 2013.

[4] T. Kim and T. Hyeon, "Applications of inorganic nanoparticles as agent," Nanotechnology, vol. 25, no. 1, Article ID 012001, 2014.

[5] A. E. Prigodich, P. S. Randeria, W. E. Briley et al., "Multiplexed nanoflares: MRNA detection in live cells," Analytical Chemistry, vol. 84, no. 4, pp. 2062-2066, 2012.

[6] D. Zhu, X. Zhou, and D. Xing, "Ultrasensitive aptamer-based bio bar code immunomagnetic separation and electrochemiluminescence method for the detection of protein," Analytica Chimica Acta, vol. 725, pp. 39-43, 2012. 
[7] D. W. Hatchett and M. Josowicz, "Composites of intrinsically conducting polymers as sensing nanomaterials," Chemical Reviews, vol. 108, pp. 746-769, 2008.

[8] D. Frank, C. Tyagi, L. Tomar et al., "Overview of the role of nanotechnological innovations in the detection and treatment of solid tumors," International Journal of Nanomedicine, vol. 9, pp. 589-613, 2014.

[9] T. Yasui, N. Kaji, and Y. Baba, "Nanobiodevices for biomolecule analysis and imaging," Annual Review of Analytical Chemistry, vol. 6, pp. 83-96, 2013.

[10] K. Seshan, Handbook of Thin-Film Deposition Processes and Techniques-Principles, Methods, Equipment and Applications, William Andrew Publishing, Noyes, Minn, USA, 2002.

[11] A. D. Maynard, R. J. Aitken, T. Butz et al., "Safe handling of nanotechnology," Nature, vol. 444, pp. 267-269, 2006.

[12] A. Nel, T. Xia, L. Mädler, and N. Li, “Toxic potential of materials at the nanolevel," Science, vol. 311, no. 5761, pp. 622-627, 2006.

[13] H. Meng, Z. Chen, G. M. Xing et al., "Ultrahigh reactivity provokes nanotoxicity: explanation of oral toxicity of nanocopper particles," Toxicology Letters, vol. 175, no. 1-3, pp. 102110, 2007.

[14] N. Singh, B. Manshian, G. J. S. Jenkins et al., "NanoGenotoxicology: the DNA damaging potential of engineered nanomaterials," Biomaterials, vol. 30, no. 23-24, pp. 3891-3914, 2009.

[15] K. W. Powers, M. Palazuelos, B. M. Moudgil, and S. M. Roberts, "Characterization of the size, shape, and state of dispersion of nanoparticles for toxicological studies," Nanotoxicology, vol. 1, no. 1, pp. 42-51, 2007.

[16] K. L. Aillon, Y. M. Xie, N. El-Gendy, C. J. Berkland, and M. L. Forrest, "Effects of nanomaterial physicochemical properties on in vivo toxicity," Advanced Drug Delivery Reviews, vol. 61, no. 6, pp. 457-466, 2009.

[17] H. Yin, H. P. Too, and G. M. Chow, "The effects of particle size and surface coating on the cytotoxicity of nickel ferrite," Biomaterials, vol. 26, no. 29, pp. 5818-5826, 2005.

[18] Y. Hu, J. Xie, Y. W. Tong, and C. Wang, "Effect of PEG conformation and particle size on the cellular uptake efficiency of nanoparticles with the HepG2 cells," Journal of Controlled Release, vol. 118, no. 1, pp. 7-17, 2007.

[19] N. Lewinski, V. Colvin, and R. Drezek, "Cytotoxicity of nanopartides," Small, vol. 4, no. 1, pp. 26-49, 2008.

[20] J. Lovrić, H. S. Bazzi, Y. Cuie, G. R. A. Fortin, F. M. Winnik, and D. Maysinger, "Differences in subcellular distribution and toxicity of green and red emitting CdTe quantum dots," Journal of Molecular Medicine, vol. 83, no. 5, pp. 377-385, 2005.

[21] P. Aggarwal, J. B. Hall, C. B. McLeland, M. A. Dobrovolskaia, and S. E. McNeil, "Nanoparticle interaction with plasma proteins as it relates to particle biodistribution, biocompatibility and therapeutic efficacy," Advanced Drug Delivery Reviews, vol. 61, no. 6, pp. 428-437, 2009.

[22] S. T. Holgate, "Exposure, uptake, distribution and toxicity of nanomaterials in humans," Journal of Biomedical Nanotechnology, vol. 6, no. 1, pp. 1-19, 2010.

[23] L. Risom, P. Møller, and S. Loft, "Oxidative stress-induced DNA damage by particulate air pollution," Mutation Research, vol. 592, no. 1-2, pp. 119-137, 2005.

[24] K. Donaldson and V. Stone, "Current hypotheses on the mechanisms of toxicity of ultrafine particles," Annali dell'Istituto Superiore di Sanita, vol. 39, no. 3, pp. 405-410, 2003.
[25] W. H. De Jong, W. I. Hagens, P. Krystek, M. C. Burger, A. J. A. M. Sips, and R. E. Geertsma, "Particle size-dependent organ distribution of gold nanoparticles after intravenous administration," Biomaterials, vol. 29, no. 12, pp. 1912-1919, 2008.

[26] J. R. Gurr, A. S. S. Wang, C. H. Chen, and K. Y. Jan, "Ultrafine titanium dioxide particles in the absence of photoactivation can induce oxidative damage to human bronchial epithelial cells," Toxicology, vol. 213, no. 1-2, pp. 66-73, 2005.

[27] B. Asgharian and O. T. Price, "Deposition of ultrafine (NANO) particles in the human lung," Inhalation Toxicology, vol. 19, no. 13, pp. 1045-1054, 2007.

[28] W. G. Kreyling, M. Semmler, F. Erbe et al., "Translocation of ultrafine insoluble iridium particles from lung epithelium to extrapulmonary organs is size dependent but very low," Journal of Toxicology and Environmental Health A, vol. 65, no. 20, pp. 1513-1530, 2002.

[29] H. J. Johnston, G. Hutchison, F. M. Christensen, S. Peters, S. Hankin, and V. Stone, "A review of the in vivo and in vitro toxicity of silver and gold particulates: particle attributes and biological mechanisms responsible for the observed toxicity," Critical Reviews in Toxicology, vol. 40, no. 4, pp. 328-346, 2010.

[30] A. Seaton and K. Donaldson, "Nanoscience, nanotoxicology, and the need to think small," The Lancet, vol. 365, no. 9463, pp. 923-924, 2005.

[31] Z. Chen, H. A. Meng, G. M. Xing et al., "Acute toxicological effects of copper nanoparticles in vivo," Toxicology Letters, vol. 163, no. 2, pp. 109-120, 2006.

[32] C. Lherm, R. H. Muller, F. Puisieux, and P. Couvreur, "Alkylcyanoacrylate drug carriers: II. Cytotoxicity of cyanoacrylate nanoparticles with different alkyl chain length," International Journal of Pharmaceutics, vol. 84, no. 1, pp. 13-22, 1992.

[33] E. J. Petersen and B. C. Nelson, "Mechanisms and measurements of nanomaterial-induced oxidative damage to DNA," Analytical and Bioanalytical Chemistry, vol. 398, no. 2, pp. 613-650, 2010.

[34] C. Ispas, D. Andreescu, A. Patel, D. V. Goia, S. Andreescu, and K. N. Wallace, "Toxicity and developmental defects of different sizes and shape nickel nanoparticles in zebrafish," Environmental Science and Technology, vol. 43, no. 16, pp. 63496356, 2009.

[35] B. D. Chithrani, A. A. Ghazani, and W. C. W. Chan, "Determining the size and shape dependence of gold nanoparticle uptake into mammalian cells," Nano Letters, vol. 6, no. 4, pp. 662-668, 2006.

[36] R. F. Hamilton Jr., N. Wu, D. Porter, M. Buford, M. Wolfarth, and A. Holian, "Particle length-dependent titanium dioxide nanomaterials toxicity and bioactivity," Particle and Fibre Toxicology, vol. 6, article 35, 2009.

[37] A. Verma and F. Stellacci, "Effect of surface properties on nanoparticle-cell interactions," Small, vol. 6, no. 1, pp. 12-21, 2010.

[38] J. A. Champion and S. Mitragotri, "Role of target geometry in phagocytosis," Proceedings of the National Academy of Sciences of the United States of America, vol. 103, no. 13, pp. 4930-4934, 2006.

[39] M. Lee, S. Lim, and C. Kim, "Preparation, characterization and in vitro cytotoxicity of paclitaxel-loaded sterically stabilized solid lipid nanoparticles," Biomaterials, vol. 28, no. 12, pp. 21372146, 2007.

[40] S. T. Kim, A. Chompoosor, Y. Yeh, S. S. Agasti, D. J. Solfiell, and V. M. Rotello, "Dendronized gold nanoparticles for siRNA delivery," Small, vol. 8, no. 21, pp. 3253-3256, 2012. 
[41] K. H. Park, M. Chhowalla, Z. Iqbal, and F. Sesti, "Single-walled carbon nanotubes are a new class of ion channel blockers," Journal of Biological Chemistry, vol. 278, no. 50, pp. 5021250216, 2003.

[42] Y. Chen, Y. Hung, I. Liau, and G. S. Huang, "Assessment of the in vivo toxicity of gold nanoparticles," Nanoscale Research Letters, vol. 4, no. 8, pp. 858-864, 2009.

[43] I. Hsiao and Y. Huang, "Effects of various physicochemical characteristics on the toxicities of $\mathrm{ZnO}$ and $\mathrm{TiO}_{2}$ nanoparticles toward human lung epithelial cells," Science of the Total Environment, vol. 409, no. 7, pp. 1219-1228, 2011.

[44] B. Fubini, I. Fenoglio, M. Tomatis, and F. Turci, "Effect of chemical composition and state of the surface on the toxic response to high aspect ratio nanomaterials," Nanomedicine, vol. 6, no. 5, pp. 899-920, 2011.

[45] M. Lippmann, "Effects of fiber characteristics on lung deposition, retention, and disease," Environmental Health Perspectives, vol. 88, pp. 311-317, 1990.

[46] A. A. Shvedova, E. R. Kisin, R. Mercer et al., "Unusual inflammatory and fibrogenic pulmonary responses to singlewalled carbon nanotubes in mice," The American Journal of Physiology_Lung Cellular and Molecular Physiology, vol. 289, no. 5, pp. L698-L708, 2005.

[47] C. A. Poland, R. Duffin, I. Kinloch et al., "Carbon nanotubes introduced into the abdominal cavity of mice show asbestoslike pathogenicity in a pilot study," Nature Nanotechnology, vol. 3, no. 7, pp. 423-428, 2008.

[48] A. Hoshino, K. Fujioka, T. Oku et al., "Physicochemical properties and cellular toxicity of nanocrystal quantum dots depend on their surface modification," Nano Letters, vol. 4, no. 11, pp. 2163-2169, 2004.

[49] A. Pietroiusti, M. Massimiani, I. Fenoglio et al., "Low doses of pristine and oxidized single-wall carbon nanotubes affect mammalian embryonic development," ACS Nano, vol. 5, no. 6, pp. 4624-4633, 2011.

[50] J. V. Georgieva, D. Kalicharan, P. Couraud et al., "Surface characteristics of nanoparticles determine their intracellular fate in and processing by human blood-brain barrier endothelial cells in vitro," Molecular Therapy, vol. 19, no. 2, pp. 318-325, 2011.

[51] C. M. Goodman, C. D. McCusker, T. Yilmaz, and V. M. Rotello, "Toxicity of gold nanoparticles functionalized with cationic and anionic side chains," Bioconjugate Chemistry, vol. 15, no. 4, pp. 897-900, 2004.

[52] T. C. K. Heiden, E. Dengler, W. J. Kao, W. Heideman, and R. E. Peterson, "Developmental toxicity of low generation PAMAM dendrimers in zebrafish," Toxicology and Applied Pharmacology, vol. 225, no. 1, pp. 70-79, 2007.

[53] S. Bhattacharjee, L. H. J. D. Haan, N. M. Evers et al., "Role of surface charge and oxidative stress in cytotoxicity of organic monolayer-coated silicon nanoparticles towards macrophage NR8383 cells," Particle and Fibre Toxicology, vol. 7, no. article 25, 2010.

[54] A. K. Kohli and H. O. Alpar, "Potential use of nanoparticles for transcutaneous vaccine delivery: effect of particle size and charge," International Journal of Pharmaceutics, vol. 275, no. 12, pp. 13-17, 2004.

[55] R. J. Griffitt, J. Luo, J. Gao, J. Bonzongo, and D. S. Barber, "Effects of particle composition and species on toxicity of metallic nanomaterials in aquatic organisms," Environmental Toxicology and Chemistry, vol. 27, no. 9, pp. 1972-1978, 2008.
[56] H. Zhang, B. Gilbert, F. Huang, and J. F. Banfield, "Water-driven structure transformation in nanoparticles at room temperature," Nature, vol. 424, no. 6952, pp. 1025-1029, 2003.

[57] S. Yang, X. Wang, G. Jia et al., "Long-term accumulation and low toxicity of single-walled carbon nanotubes in intravenously exposed mice," Toxicology Letters, vol. 181, no. 3, pp. 182-189, 2008.

[58] P. Wick, P. Manser, L. K. Limbach et al., "The degree and kind of agglomeration affect carbon nanotube cytotoxicity," Toxicology Letters, vol. 168, no. 2, pp. 121-131, 2007.

[59] A. K. Gupta and M. Gupta, "Cytotoxicity suppression and cellular uptake enhancement of surface modified magnetic nanoparticles," Biomaterials, vol. 26, no. 13, pp. 1565-1573, 2005.

[60] C. M. Sayes, J. D. Fortner, W. Guo et al., "The differential cytotoxicity of water-soluble fullerenes," Nano Letters, vol. 4, no. 10, pp. 1881-1887, 2004.

[61] B. Fubini, E. Giamello, M. Volante, and V. Bolis, "Chemical functionalities at the silica surface determining its reactivity when inhaled. Formation and reactivity of surface radicals," Toxicology and Industrial Health, vol. 6, no. 6, pp. 571-598, 1990.

[62] C. Kirchner, T. Liedl, S. Kudera et al., "Cytotoxicity of colloidal CdSe and CdSe/ZnS nanoparticles," Nano Letters, vol. 5, no. 2, pp. 331-338, 2005.

[63] T. Kuo, C. Lee, S. Lin, C. Dong, C. Chen, and H. Tan, "Studies of intracorneal distribution and cytotoxicity of quantum dots: risk assessment of eye exposure," Chemical Research in Toxicology, vol. 24, no. 2, pp. 253-261, 2011.

[64] G. Guo, W. Liu, J. Liang, Z. He, H. Xu, and X. Yang, "Probing the cytotoxicity of CdSe quantum dots with surface modification," Materials Letters, vol. 61, no. 8-9, pp. 1641-1644, 2007.

[65] M. C. Mancini, B. A. Kairdolf, A. M. Smith, and S. Nie, “Oxidative quenching and degradation of polymer-encapsulated quantum dots: new insights into the long-term fate and toxicity of nanocrystals in vivo," Journal of the American Chemical Society, vol. 130, no. 33, pp. 10836-10837, 2008.

[66] F. Chen and D. Gerion, "Fluorescent CdSe/ZnS nanocrystalpeptide conjugates for long-term, nontoxic imaging and nuclear targeting in living cells," Nano Letters, vol. 4, no. 10, pp. 18271832, 2004.

[67] M. Mahmoudi, A. S. Milani, and P. Stroeve, "Synthesis, surface architecture and biological response of superparamagnetic iron oxide nanoparticles for application in drug delivery: a review," International Journal of Biomedical Nanoscience and Nanotechnology, vol. 1, pp. 164-201, 2010.

[68] J. Bang, B. Chon, N. Won, J. Nam, T. Joo, and S. Kim, "Spectral switching of type-II quantum dots by charging," Journal of Physical Chemistry C, vol. 113, no. 16, pp. 6320-6323, 2009.

[69] D. Dorfs, T. Franzi, R. Osovsky et al., "Type-I and typeII nanoscale heterostructures based on CdTe nanocrystals: a comparative study," Small, vol. 4, no. 8, pp. 1148-1152, 2008.

[70] L. Lacerda, A. Soundararajan, R. Singh et al., "Dynamic imaging of functionalized multi-walled carbon nanotube systemic circulation and urinary excretion," Advanced Materials, vol. 20, no. 2, pp. 225-230, 2008.

[71] M. C. Morris, E. Gros, G. Aldrian-Herrada et al., "A noncovalent peptide-based carrier for in vivo delivery of DNA mimics," Nucleic Acids Research, vol. 35, no. 7, p. e49, 2007.

[72] E. E. Connor, J. Mwamuka, A. Gole, C. J. Murphy, and M. D. Wyatt, "Gold nanoparticles are taken up by human cells but do not cause acute cytotoxicity," Small, vol. 1, no. 3, pp. 325-327, 2005. 
[73] A. E. Nel, L. Mädler, D. Velegol et al., "Understanding biophysicochemical interactions at the nano-bio interface," Nature Materials, vol. 8, no. 7, pp. 543-557, 2009.

[74] Y. S. Lin and C. L. Haynes, "Impacts of mesoporous silica nanoparticle size, pore ordering, and pore integrity on hemolytic activity," Journal of the American Chemical Society, vol. 132, no. 13, pp. 4834-4842, 2010.

[75] F. de Angelis, A. Pujia, C. Falcone et al., "Water soluble nanoporous nanoparticle for in vivo targeted drug delivery and controlled release in B cells tumor context," Nanoscale, vol. 2, no. 10, pp. 2230-2236, 2010.

[76] J. H. Park, L. Gu, G. von Maltzahn, E. Ruoslahti, S. N. Bhatia, and M. J. Sailor, "Biodegradable luminescent porous silicon nanoparticles for in vivo applications," Nature Materials, vol. 8, no. 4, pp. 331-336, 2009.

[77] T. M. Sager, D. W. Porter, V. A. Robinson, W. G. Lindsley, D. E. Schwegler-Berry, and V. Castranova, "Improved method to disperse nanoparticles for in vitro and in vivo investigation of toxicity," Nanotoxicology, vol. 1, no. 2, pp. 118-129, 2007.

[78] J. Jiang, G. Oberdörster, and P. Biswas, "Characterization of size, surface charge, and agglomeration state of nanoparticle dispersions for toxicological studies," Journal of Nanoparticle Research, vol. 11, no. 1, pp. 77-89, 2009.

[79] V. L. Colvin, "The potential environmental impact of engineered nanomaterials," Nature Biotechnology, vol. 21, no. 10, pp. 1166-1170, 2003.

[80] W. Hou, P. Westerhoff, and J. D. Posner, "Biological accumulation of engineered nanomaterials: a review of current knowledge," Environmental Sciences: Processes and Impacts, vol. 15, no. 1, pp. 103-122, 2013. 

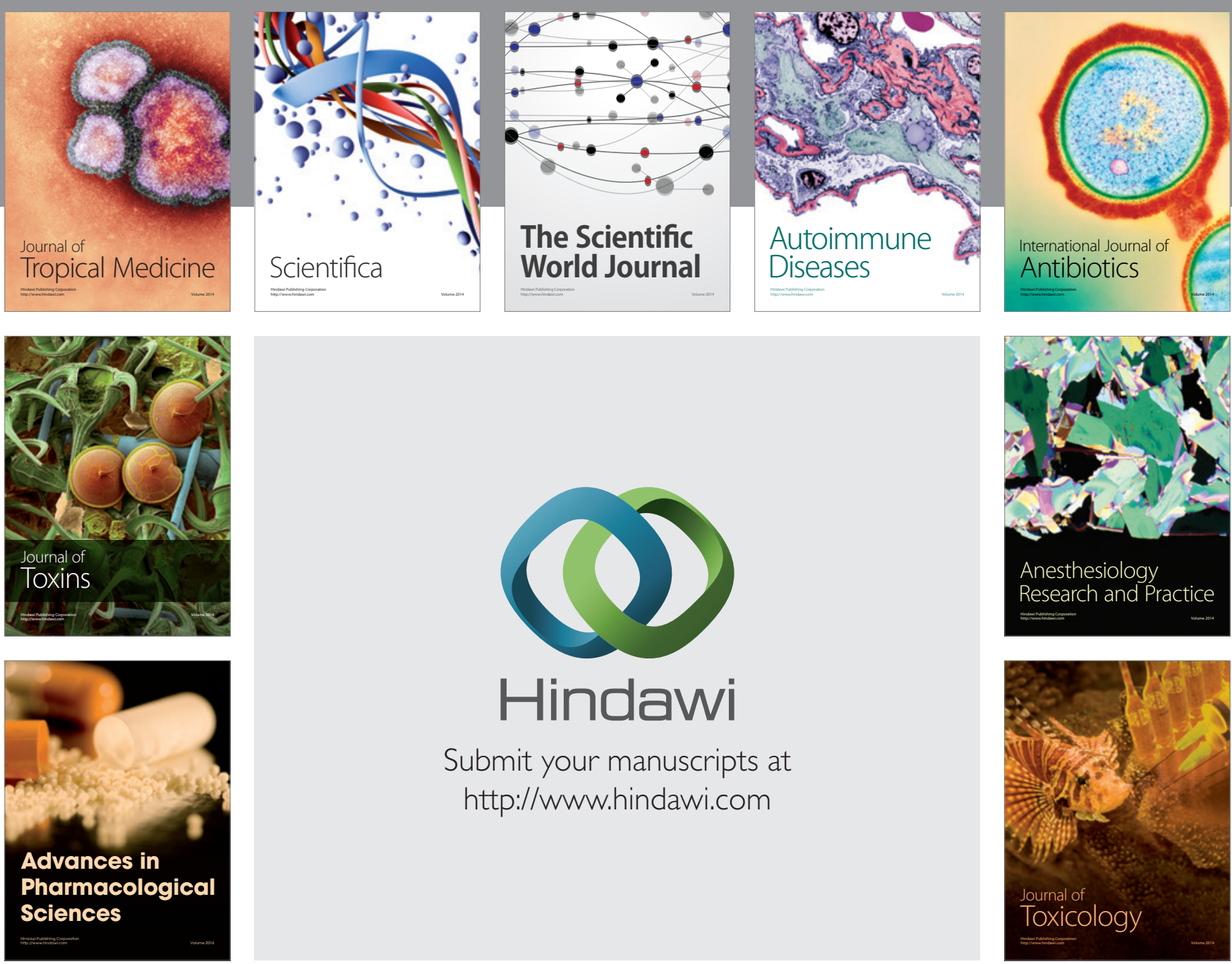

\section{Hindawi}

Submit your manuscripts at

http://www.hindawi.com
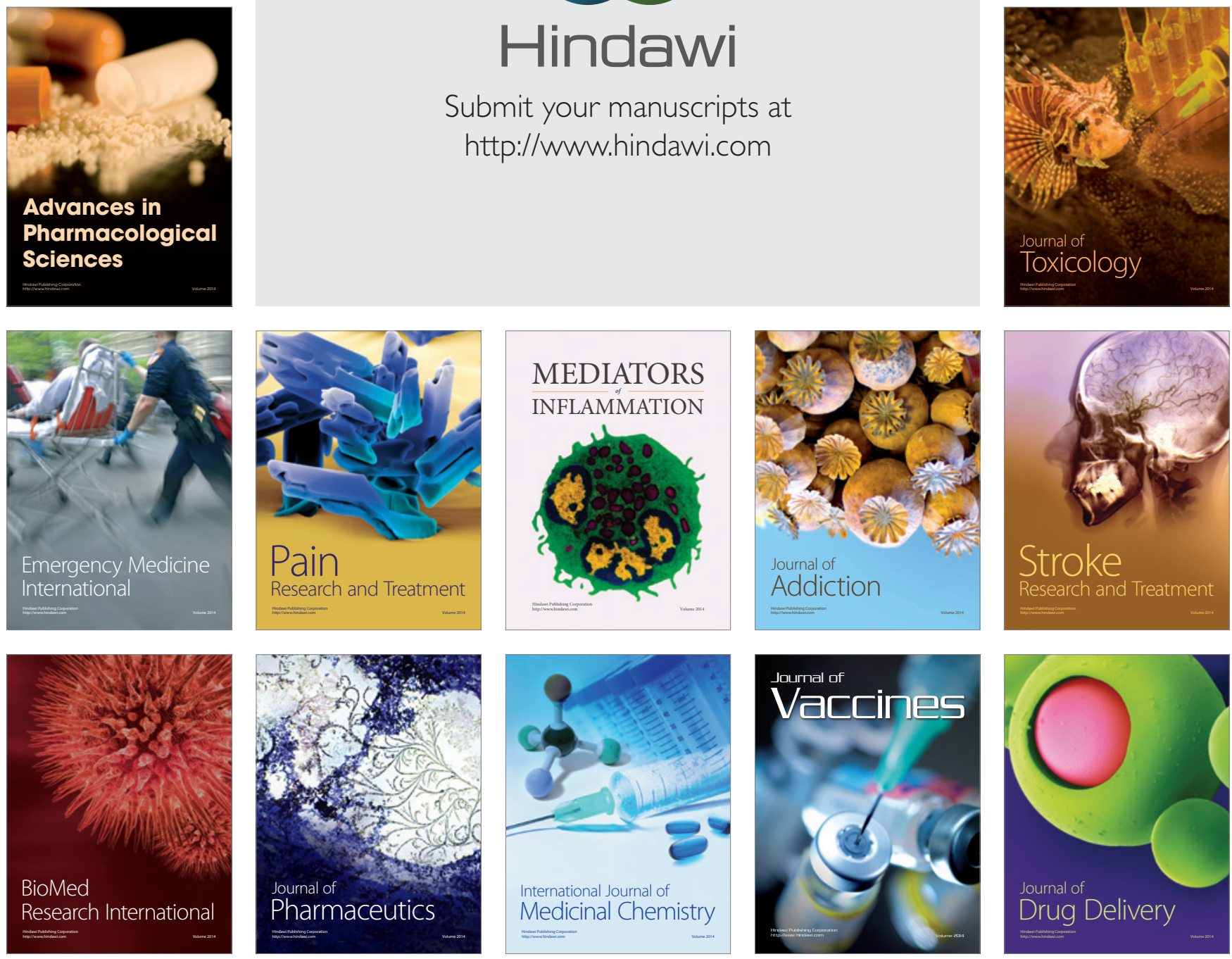